\title{
Gene socialization: gene order, GC content and gene silencing in
} Salmonella

\author{
Nikolas Papanikolaou ${ }^{1}$, Kalliopi Trachana ${ }^{2}$, Theodosios Theodosiou ${ }^{3}$, \\ Vasilis J Promponas*4 and Ioannis Iliopoulos*1
}

Address: ${ }^{1}$ Division of Medical Sciences, University of Crete Medical School, Heraklion 71110, Crete, Greece, ${ }^{2} \mathrm{EMBL}, \mathrm{Meyerhofstrasse} \mathrm{1,} \mathrm{69117}$ Heidelberg, Germany, ${ }^{3}$ Department of Informatics, School of Natural Sciences, Aristotle University of Thessaloniki, Thessaloniki, Greece and ${ }^{4}$ Bioinformatics Research Laboratory, Department of Biological Sciences, University of Cyprus, CY 1678, Nicosia, Cyprus

Email: Nikolas Papanikolaou - papnikol@med.uoc.gr; Kalliopi Trachana - trachana@embl.de; Theodosios Theodosiou - theodos@csd.auth.gr; Vasilis J Promponas* - vasilis.promponas@gmail.com; Ioannis Iliopoulos* - ioannis@iti.gr

* Corresponding authors

Published: II December 2009

BMC Genomics 2009, 10:597 doi:10.1/86/|47|-2/64-10-597
Received: 10 June 2009

Accepted: II December 2009

This article is available from: http://www.biomedcentral.com/I47I-2/64/I0/597

(c) 2009 Papanikolaou et al; licensee BioMed Central Ltd.

This is an Open Access article distributed under the terms of the Creative Commons Attribution License (http://creativecommons.org/licenses/by/2.0), which permits unrestricted use, distribution, and reproduction in any medium, provided the original work is properly cited.

\begin{abstract}
Background: Genes of conserved order in bacterial genomes tend to evolve slower than genes whose order is not conserved. In addition, genes with a GC content lower than the GC content of the resident genome are known to be selectively silenced by the histone-like nucleoid structuring protein (H-NS) in Salmonella.

Results: In this study, we use a comparative genomics approach to demonstrate that in Salmonella, genes whose order is not conserved (or genes without homologs) in closely related bacteria possess a significantly lower average GC content in comparison to genes that preserve their relative position in the genome. Moreover, these genes are more frequently targeted by $\mathrm{H}-\mathrm{NS}$ than genes that have conserved their genomic neighborhood. We also observed that duplicated genes that do not preserve their genomic neighborhood are, on average, under less selective pressure.

Conclusions: We establish a strong association between gene order, GC content and gene silencing in a model bacterial species. This analysis suggests that genes that are not under strong selective pressure (evolve faster than others) in Salmonella tend to accumulate more AT-rich mutations and are eventually silenced by H-NS. Our findings may establish new approaches for a better understanding of bacterial genome evolution and function, using information from functional and comparative genomics.
\end{abstract}

\section{Background}

The conservation of gene order has been found to play an essential role in genome evolution. More specifically, proteins encoded by genes of conserved order in bacteria tend to evolve more slowly when compared to proteins encoded by genes without a conserved order $[1,2]$ and genes with similar or related functions tend to occur in adjacent chromosomal positions in yeast [3]. Moreover, genes with conserved order were found to evolve at similar rates [4] and, in prokaryotes, proteins encoded by genes with conserved order appear to interact physically [1]. It has also been shown that in eukaryotes essential genes are clustered in regions with low recombination rates [5], whereas in bacteria essential genes are more conserved than non essential genes [6]. In addition, it has been reported that the number of interactions involved in 
a protein network is directly correlated with the rate of evolution among these proteins [7] and that highly expressed genes evolve slowly $[8,9]$. A case in point for the role of gene order in evolution can be illustrated by duplicated genes [10]. Following a duplication event one of the two (paralog) genes might keep its original function, whereas the other one might be under less selective pressure. Yet, it is not always readily apparent which duplicated genes evolve faster. There have been reports that have marginally correlated sequence conservation with genome context [11], but there must be other, yet unknown, functional features that determine the fate of duplicate genes.

Recently, two research groups observed independently that the histone-like nucleoid structuring protein (H-NS) plays an important role as a general transcriptional repressor of a large number of genes in Salmonella enterica serovar Typhimurium LT2 (S. Typhimurium) $[12,13]$. H-NS is a protein that is believed to play an essential role in the organization and compaction of bacterial chromatin as well as in transcriptional regulation for many bacterial genes [14-16]. H-NS binds to these genes and silences them transcriptionally. An apparent common feature of genes silenced by H-NS is that their GC content is significantly lower than the overall GC content of the Salmonella genome [13]. Additionally, a large proportion of these $\mathrm{H}$ NS repressed genes is predicted to have been acquired from a foreign source (horizontally transferred genes, HTGs) $[13,15]$, a fact in agreement with the observation that HTGs are relatively AT-rich $[17,18]$. It has been suggested that this may be a defensive mechanism against foreign genetic material without loosing the benefit of future usage of this material if necessary [15].

Herein, we aimed to test the correlation between gene order conservation, gene duplication and H-NS dependent silencing in S. Typhimurium, using Escherichia coli K12 as a reference genome in order to identify the conservation or loss of gene order along the bacterial chromosome. We have also attempted to associate the above mentioned features with GC content and gene essentiality.

\section{Results \\ Evolutionary rate and GC content are related to genomic neighborhood conservation}

We compared the proteins predicted to be encoded in the completely sequenced genome of S. Typhimurium [19] against the proteins encoded in the genome of $E$. coli K12 [20], both obtained from the NCBI Genomes Division ftp://ftp.ncbi.nih.gov/genomes/Bacteria/ using BLASTP [21]. Using the criteria described in Materials and Methods, we identify 3584 homologs (out of 4425 Salmonella proteins in total) between these two highly related bacte- rial species, of which 3024 were found to be encoded by genes of conserved order (GCO) and 560 proteins encoded by genes that have lost their order (nGCO) (Additional file 1). The remaining 841 genes either fell below the E-value threshold or exhibited significant similarities only in short segments, when compared to their full length sequences and thus were not considered to have a homolog in E. coli K12. The 3024 homolog pairs with conserved gene order share an average sequence identity of $83.6 \%$, whereas the 560 proteins that were encoded by genes with no order conservation share an average sequence identity of $45.0 \%$ (Fig. 1A). The difference between the two groups is statistically significant according to a Wilcoxon rank-sum test (W $=1522518, \mathrm{P}$ value $<2.2 e-16)$. Subsequent analysis revealed statistically significant differences in the GC content for the three aforementioned classes of Salmonella genes (GCO, nGCO, without an E. coli K12 homolog), which was 53.7\%, $50.6 \%$ and $49.2 \%$ respectively (Fig. 1B). Taking into account that the GC content of the Salmonella genome is $52.2 \%$ it is readily apparent that GCO are enriched in Gs and Cs. This sizable decrease in GC content in the last two groups could be explained either by the presence of a large number of horizontally transferred genes in the last two groups, or by an accumulation of AT-rich mutations in genes belonging to these groups.

In order to check the former hypothesis, we cross-checked the three groups for the existence of HTGs in the Salmonella genome using HGT-DB, a database of putative horizontally transferred genes in prokaryotes [22]. As expected, the majority of predicted HTGs fall into the last two categories (nGCO, no homolog), but after removing HTGs from the complete gene set, the results were not affected (Additional file 2). However, this observation could be biased because the methodological approach used in HGT-DB to infer lateral gene transfer events, is largely dependent on GC content [22]. The latter hypothesis could be explained by our finding that the last two categories of genes may be under weaker selective pressure than the first one and, consequently, accumulating a larger number of mutations, especially C-to-T mutations which are known to be the most common ones under neutral evolution [23]. The observation that genomes of intracellular pathogens tend to be AT rich for energetic and resources availability reasons [24] could also explain the aforementioned result. After a speciation event occurs, some genes might remain GCO and continue to evolve slowly. This is probably the result of the strong evolutionary pressure to maintain their relative order, assisting the preservation of protein function or interaction with neighboring genes. On the other hand, other genes might lose their relative genomic position and start evolving faster. Genes in Salmonella with no homologs in E. coli K12 could either be HTGs or genes that have lost their 

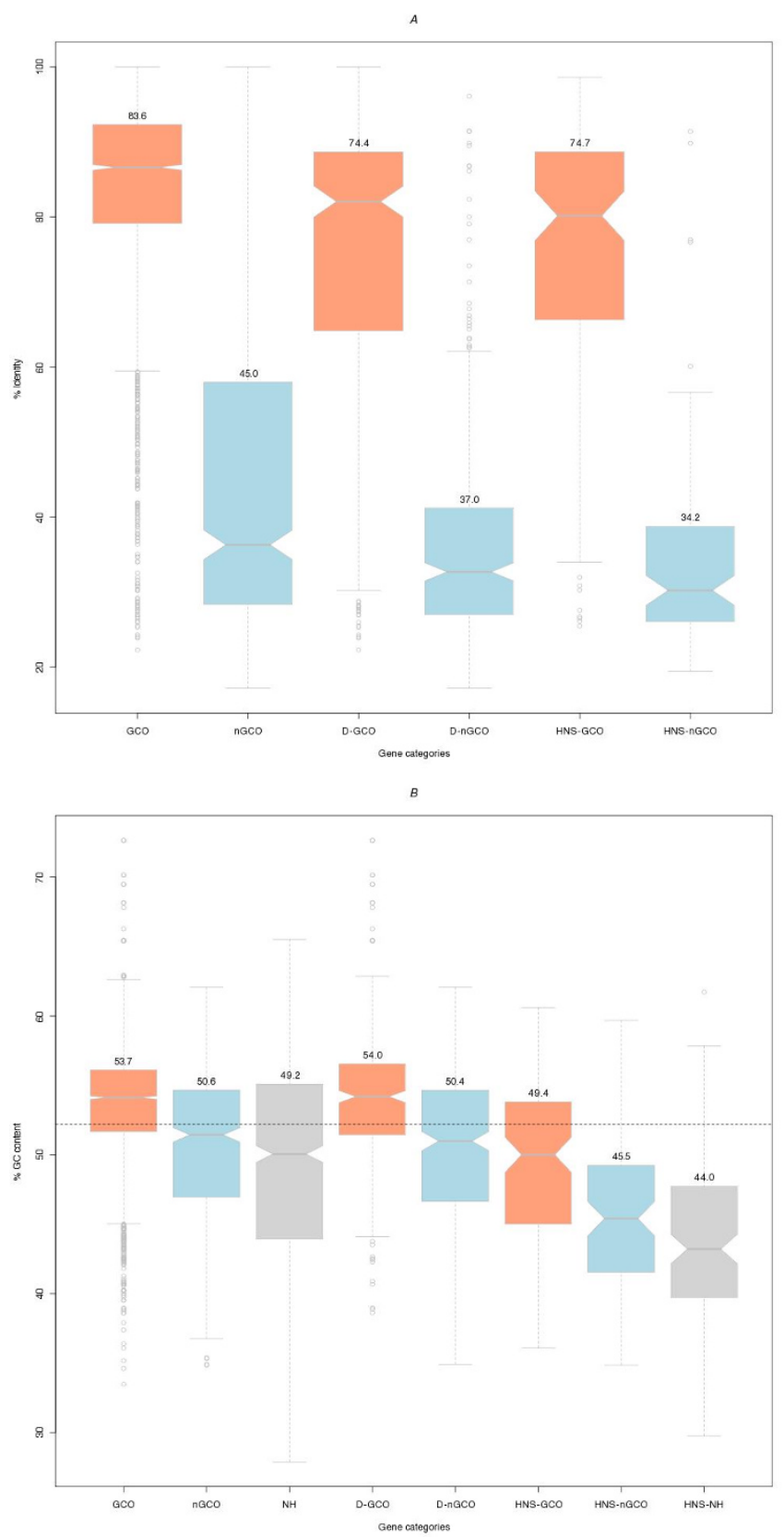

Figure I

Correlation of gene order conservation with sequence identity and GC content (Salmonella vs E. coli KI2). (A) Sequence identity frequency distributions of proteins encoded by GCO/nGCO genes for datasets used in this study. Boxandwhisker plots illustrate the differences between the medians and the dispersion of the respective datasets. Orange and light blue represent GCO versus nGCO datasets. Average values are displayed on top of the box plots. The two leftmost box plots (GCO, nGCO) depict differences between those two gene classes within the overall protein sequence data set. The next two data sets depict differences between duplicated GCO genes (DGCO) and duplicated nGCO genes (DnGCO). The last two datasets represent HNS repressed genes (HNSGCO: HNS repressed GCO genes, HNSnGCO: HNS repressed nGCO genes). (B) GC content of GCO genes, nGCO genes and genes with no homolog in $E$. coli $\mathrm{KI} 2 \mathrm{NH}$ for datasets used in this study. Additionally to the coloring scheme of Fig. IA, we use light grey for sequences that had no homolog in $E$. coli $\mathrm{K} / 2$. The dashed horizontal line corresponds to the overall GC content of S. Typhimurium genome (52.2\%). (For a more detailed description, including statistical analysis see Additional files 8, 9). 
genomic neighborhood and subsequently evolved to such an extent that no significant sequence similarities could be detected to support homology to any E. coli K12 gene.

In order to obtain a quantitative estimate of the selective constraint for the groups of GCO and nGCO genes we calculated the ratio of non-synonymous (Ka) to synonymous (Ks) nucleotide substitutions by projecting the protein sequence alignments to the respective genomic sequences. The mean Ka/Ks ratio for GCO and nGCO groups was calculated to be 0.07 and 0.23 respectively, using the yn00 method [25] as implemented in the PAML package [26] (Fig. 2). This difference is found to be statistically significant and is indicative of a stronger selective constraint under which GCO genes are evolving in Salmonella.

\section{The fate of duplicated genes is determined by gene order conservation}

The most striking evidence that GCO genes evolve, on average, significantly slower than the nGCO genes, is inferred from an analysis of the subset of duplicated genes. We performed an all-against-all self-comparison of proteins encoded in the Salmonella genome and detected all groups of genes that could be assigned as homologs in the Salmonella genome that share the same best hit in the E. coli K12 genome (Additional file 3). We identified 687 such genes (15.5\% of the complete Salmonella gene set) 343 of which are GCO (49.9\%) and 344 are nGCO $(51.1 \%)$. When compared to the same figures for the complete Salmonella gene set ( $68.3 \%$ and $12.6 \%$ respectively) the enrichment of the duplicate gene set in nGCO genes is evident. This enrichment probably takes place because, after a duplication event occurs, the selective pressure on one gene to retain its genomic neighborhood becomes less stringent. The proteins of GCO genes in the 343 duplicates share a $74.4 \%$ identity (standard deviation sd = 20.2), whereas for the 344 proteins of nGCO genes this figure drops to $37.0 \%$ ( $\mathrm{sd}=14.6$; Wilcoxon rank-sum test: $\mathrm{W}=106315.5$, P-value $=0)($ Fig. 1A). This result leads us to two major conclusions: First, as it may be deduced from a comparison of these identity percentages with the result for the complete genome set, the proteins encoded by this subset of duplicated genes evolve faster (in agreement with previous studies [27]). Second, whenever a gene duplication event occurs, the gene that retains its gene order tends to evolve more slowly than the one that travels away from the ancestral gene neighborhood and probably it is the former that keeps the original function. This finding complements an earlier study [11], although different experimental approaches have been employed here. Our results, even though based on the average conservation of putative homologs, corroborate the finding that genomic neighborhood conservation is an important additional criterion that should be taken into account in protein sequence similarity searches. We reached the same conclusions after calculating the $\mathrm{Ka} / \mathrm{Ks}$ ratio for the two different duplicated gene groups - GCO and nGCO - with respective values 0.09 and 0.43 (Fig. 2). In most cases where all duplicated genes were nGCO, none of the genes exhibited particularly high identity to the common E. coli K12 homolog (Additional file 4). An analysis of the average GC content for the duplicated genes (GCO and nGCO) showed a similar pattern with the one of the overall genome (Fig. 1B).

\section{Essential genes tend to conserve their order}

Further examination showed that, of the 231 experimentally verified Salmonella genes that were found to be essential in a recent study [28], 184 were GCO $(79.6 \%$ compared to $68.3 \%$ for the overall genome representation), 27 were nGCO (almost equal representation 11.7\% compared to $12.6 \%$ ) and 20 were without an E. coli K12 homolog (8.6\% compared to $19.0 \%$, see also Fig. 3$)$. To support our finding, we generated 1000 random sets of 231 genes (sampled without replacement from the Salmonella genome) and examined the distributions of genes belonging to the three different classes: $68.4 \%, 12.6 \%$, $18.9 \%$ respectively, very close to the values for the complete gene set (Fig. 3). The 211 essential genes with an $E$. coli $\mathrm{K} 12$ homolog encode for proteins that exhibit average sequence identity of $82.5 \%$ ( $\mathrm{sd}=19.8$ ). These results show that essential genes in bacteria are not only more evolutionary conserved than non essential ones, as was previously shown [6], but they also tend to be GCO, at least in Salmonella.

\section{H-NS preferentially regulates genes that do not conserve their order}

Driven by recent results demonstrating that H-NS silences a significant amount of genes thought to have been acquired by lateral gene transfer, we calculated the number of GCO and nGCO genes in the dataset of 359 genes that were found to be silenced by the action of $\mathrm{H}$ NS in the Salmonella chromosome [13]. We ended up with the following numbers: 119 genes were GCO (33.1\%), 99 $(27.6 \%)$ were nGCO and $141(39.3 \%)$ were without any detectable homolog in E. coli K12. The difference in the gene order status of genes compared to the overall genome is striking, with the representation being $68.3 \%$, $12.6 \%$ and $19 \%$ respectively (see also fig. 3 ). This result demonstrates a preference of H-NS to repress genes that are either nGCO or that have no homologs in closely related species. The 119 GCO genes shared $74.7 \%$ identity and the nGCO $34.2 \%$ (P-value $<2.2 \mathrm{e}-16$ ), a finding consistent with the results for both the overall genome set and the set of duplicated genes (Fig. 1A).

We calculated the GC content for the H-NS repressed GCO genes (49.4\%), nGCO genes (45.5\%) and genes with no detected homolog in E. coli K12 (44.0\%), which 


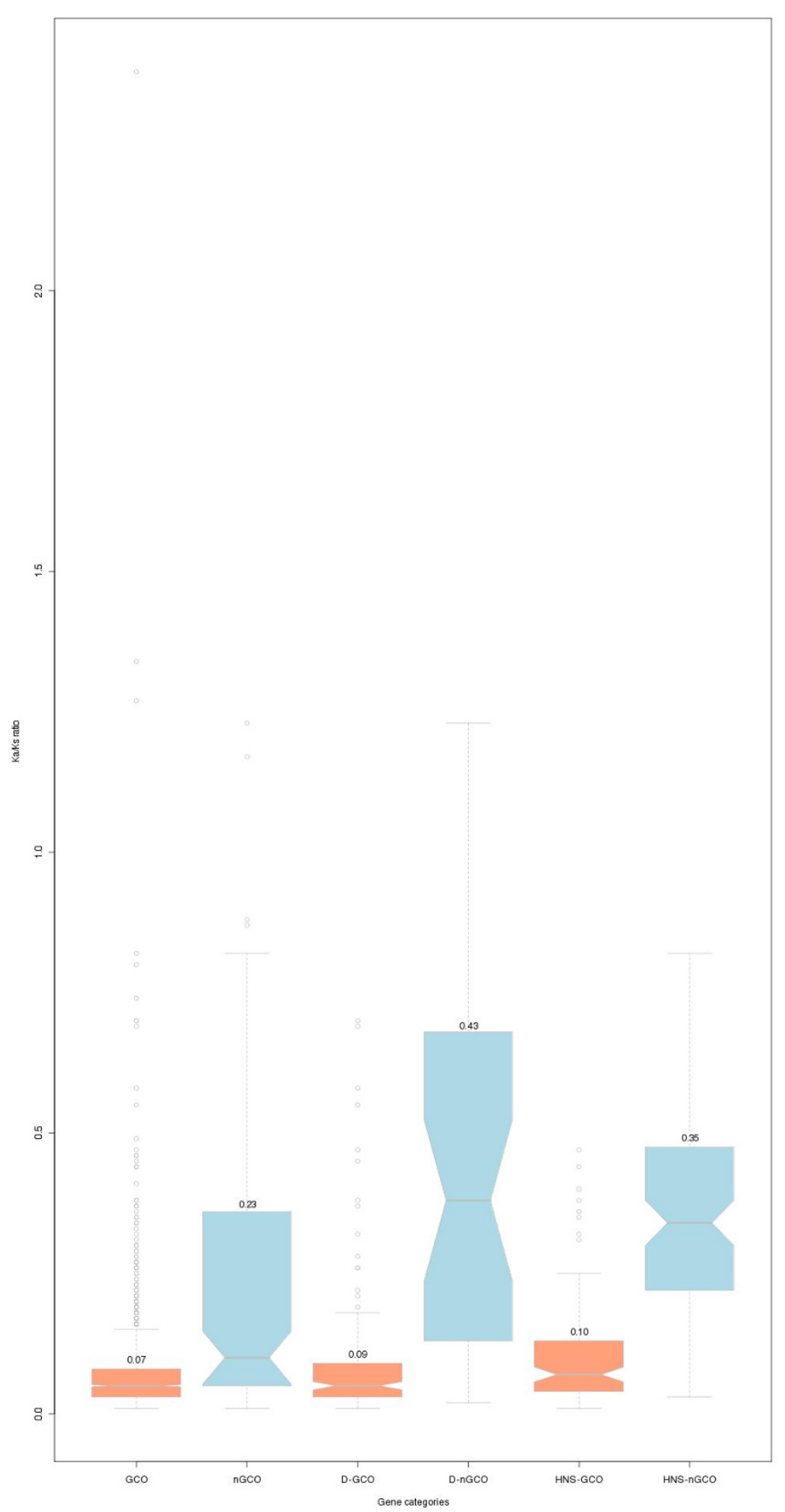

Figure 2

Boxandwhisker plots of $\mathrm{Ka} / \mathrm{Ks}$ ratio distributions for $\mathrm{GCO}$ and $\mathrm{nGCO}$ genes for various datasets used in this study. Significant differences in $\mathrm{Ka} / \mathrm{Ks}$ ratios were observed between GCO and nGCO genes both in the overall dataset (Wilcoxon ranksum test: $\mathrm{W}=2033$ I3, Pvalue $=0$, standard deviations 0.09 and 0.18 respectively) and in the subset of duplicated genes (DGCO: Duplicated GCO genes, DnGCO: Duplicated nGCO genes. $W=13595.5$, Pvalue $=0$, standard deviations 0.13 and 0.17 respectively). The same coloring scheme with Fig. IA. is used for easy comparison. 


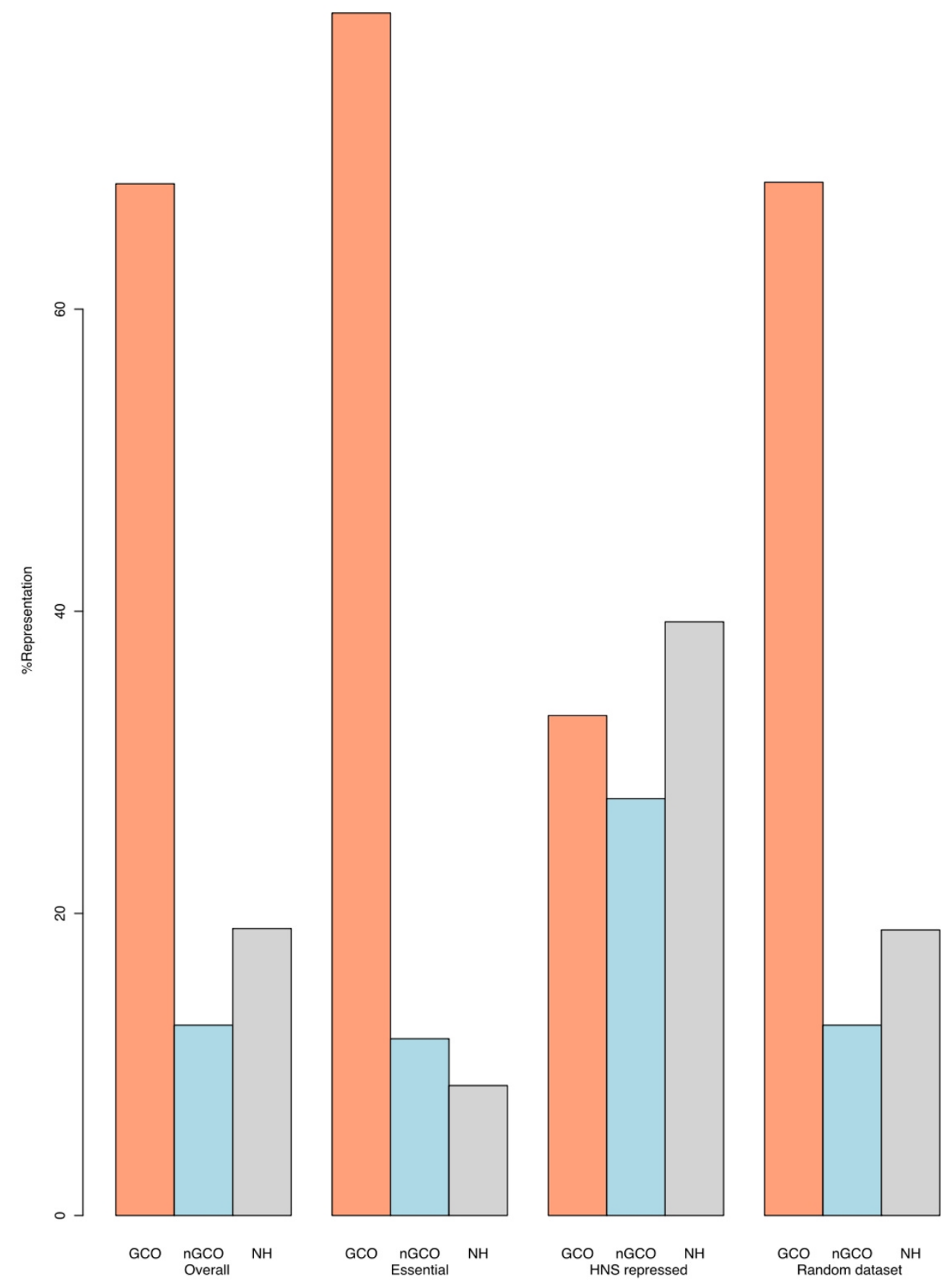

Figure 3

Distribution of the three categories of genes (GCO, nGCO and genes with no homolog in E. coli) in the complete Salmonella gene set and in the subsets of HNS repressed genes [13] and essential genes [28]. GCO genes are overrepresented in the essential gene set and underrepresented in HNS repressed genes. In contrary, nGCO genes and genes with no homolog in E. coli are overrepresented in HNS repressed genes. As expected, we observed that genes with no homolog in $E$. coli are underrepresented in the essential gene set. In the random dataset we observe the same representation as in the overall Salmonella genome. The same coloring scheme with Fig. IB. is used for easy comparison. 
is a significant decrease of the GC content for all three different groups of genes (P-value < 5.498e-12) (Fig. 1B). Using a list of putative HTGs [22] we identified 130 genes out of the $359 \mathrm{H}-\mathrm{NS}$ repressed Salmonella genes that were predicted to be horizontally transferred. Although there are many genes from the 359 which are predicted to be HTGs - as previously reported [13] - there is still a large number of H-NS repressed genes that were not predicted to be horizontally transferred. An interesting finding was that in the set of $229 \mathrm{H}-\mathrm{NS}$ repressed genes (not predicted to be HTGs) presented a higher GC content compared to the $130 \mathrm{H}$-NS repressed genes predicted to be HTGs (Table 1 and Additional file 1). Someone may argue that these results may be biased due to the method followed in [22]. However, we observed a similar pattern of decrease in GC content compared to the one calculated for the complete Salmonella gene set (Table 1).

In order to clarify whether the observed differences are due to pathogenicity related genes, we also compared Salmonella with an enteropathogenic strain of $E$. coli (O127:H6 str. E2348/69) (EPEC) [29] ftp.ncbi.nih.gov/genomes/Bacteria/. We identified the complement of genes that had no homolog between Salmonella and E. coli K12 and had a homolog between Salmonella and EPEC (Additional file 5). Among the H-NS repressed genes within this dataset (14 genes out of 174 in total), we detected genes of Salmonella Pathogenicity Island-2 (SPI-2) such as ssaL, $S$ [30], sseB and sscA [31]. We also identified Salmonella genes whose order switches from nGCO to GCO when the reference genome is K12 and EPEC, respectively (Additional file 6). In this dataset, the H-NS repressed genes (11 genes out of 55 in total) include genes of SPI-2 such as ssaV, N, R, T, U [30].

Another interesting observation in the 359 H-NS repressed genes was the identification of 98 duplicated genes within this dataset $(27.3 \%$ compared to $15.5 \%$ of duplicated genes in the whole genome) out of which only
29 were GCO. We subsequently analyzed the GC content for the duplicated genes of which only one paralog is targeted by H-NS. Of the 98 duplicated genes, a subset of 74 (out of which 22 were GCO) were identified to have 89 paralogs which are not H-NS repressed. After comparing the GC content of the above subsets of paralogs, we observed a striking difference. More specifically, the GC content of the $74 \mathrm{H}-\mathrm{NS}$ repressed genes was $46.5 \%$ (sd = 5.5) and for their 89 paralogs the GC content was $51.4 \%$ ( $\mathrm{sd}=4.2$ ) (Wilcoxon rank-sum test: $\mathrm{W}=1545, \mathrm{P}$-value $=$ $5.663 e-09)$. The above results provide a strong indication that, apart from repressing horizontally transferred genes, H-NS may also play a role in the transcriptional regulation of duplicated genes. Interestingly, genes that play an important role in the pathogenicity of Salmonella such as ssrB (essential for the expression of SPI-2 genes [32] genes) and ssaN, R, T, U and V (genes of SPI-2 that encode components of the second type III secretion apparatus [30]) were found to be nGCO, with low GC content (43.1 average GC\%) whereas their corresponding duplicates were GCO with significantly higher GC content (54.79 average $\mathrm{GC} \%$ ). Moreover, the aforementioned virulence associated genes were identified to be repressed by H-NS [13], whereas their duplicates' expression was not significantly affected by H-NS.

Surprisingly, 20 genes out of those 359 were found to be essential for Salmonella. This paradox may be explained either by the growth conditions under which experiments where carried out [28] or by introducing H-NS as a part of a general, yet currently unknown, bacterial regulatory mechanism.

\section{Discussion}

Taken together, this work shows that in Salmonella: (a) the proteins encoded by GCO genes tend to be more conserved (Fig. 1A and Fig. 2). Probably this is due to a correlation of conservation of gene order and the number of interacting proteins and/or expression levels of proteins

Table I: Gene order conservation of HNS repressed genes not predicted to be HTGs is associated to their GC content.

\begin{tabular}{lll}
\hline & HNSnonHTGs & \\
\hline Gene order status & Number of Genes (\%) & Average \%GC content (sd) \\
\hline GCO & $93(40.6)$ & $51.2(4.8)$ \\
\hline nGCO & $54(23.6)$ & $47.6(5.3)$ \\
\hline No E. coli KI2 homolog & $82(35.8)$ & $45.4(6.7)$ \\
\hline
\end{tabular}

We illustrate features of the subset of 229 HNS repressed genes predicted not to be HTGs (HNSnonHTGs). More specifically, the distribution of HNSnonHGTs genes in the three categories depending on gene order conservation largely deviates from the overall genome representation. GCO genes are still underrepresented but significantly increased compared to predicted HTGs (see additional file 10). However, we observe the same characteristic decrease in the average GC content between these gene sets with the overall genome (c.f. Fig. IB) (KruskalWallis chisquared = 37.8234, $\mathrm{df}=2$, Pvalue $<6.12 \mathrm{e} 09$ ). 
encoded by genes belonging to the conserved clusters. Although it is known that protein expression levels are correlated to protein evolutionary rates $[8,9]$, and it is rather controversial whether there is a correlation between the connectivity of nodes in protein interaction networks and evolutionary rate $[2,7,33-35]$, it still remains to be shown if any of these findings correlate to gene order conservation as well. (b) GCO genes have on average higher GC content when compared to genes that are nGCO and even higher than genes that do not share any homology to E. coli K12 genes (Fig. 1B). We speculate that this is a result of a mechanism that leads genes which evolve faster than others (and are not under strong selective pressure) to accumulate AT-rich mutations. (c) Genes that remain GCO have on average significantly lower $\mathrm{Ka} / \mathrm{Ks}$ ratio than the nGCO ones, indicating that they are under higher selective pressure (Fig. 2). (d) Whenever a duplication event takes place, the duplicate that keeps its original order, is the one that keeps the highest sequence similarity (at least on average) and possibly the original function (in agreement to [11]). On the other hand, when the duplicated gene does not preserve its original order, it is subject to much faster evolution and less selective pressure as it is shown by comparing the respective $\mathrm{Ka} / \mathrm{Ks}$ ratios (Fig. 2). Overrepresentation of nGCO genes in the duplicated group demonstrates that selective pressure on genes to keep their order is more relaxed after a duplication event. Interestingly, after a duplication event occurs and none of the paralogs retain their ancestral position in the genome, we failed to find any preference for sequence conservation (Additional file 4). (e) Finally, we demonstrated that the silencing mechanism via the H-NS protein in Salmonella significantly affects genes that are nGCO or genes that do not have any (detectable) homolog in E. coli K12. This could be another indication that H-NS might play a role, not only as a defensive instrument against foreign acquired DNA $[12,13,36]$ but also as an internal control of native genes [15], as it could be of benefit to the organism to have them repressed for a variety of reasons associated with genome structure, for duplicated genes, nonessential genes or genes that did not retain their order.

\section{Conclusions}

In our analysis we tried to correlate gene order conservation with gene duplication, GC content and gene silencing in Salmonella enterica serovar Typhimurium LT2 based on two publications concerning H-NS silencing in the aforementioned species. We found that genes of conserved order not only evolve slower, as previously reported, but also tend to have higher GC content than the ones that do not conserve their order. We also found that essential genes tend to retain their genome position. Another finding was that after a duplication event takes place the gene that keeps its original position evolves slower than the one that looses it and probably keeps its primary function.
Our study also shows that H-NS protein, a general repressor of a large number of genes in Salmonella, tends to avoid binding on genes with a conserved order. There is a clear indication that from the duplicated genes H-NS "prefers" to repress those ones that do not have a conserved gene order.

\section{Methods \\ Definition of genes of conserved order}

As a measure of conservation at the amino acid sequence level, we used the percentage of identical residues reported by BLASTP, while disregarding conservative substitutions and gaps. We follow the same criteria of gene order conservation as in [2]. More specifically, given two genomes $\mathrm{A}$ and $\mathrm{B}$ encoding $\mathrm{N}_{\mathrm{a}}$ and $\mathrm{N}_{\mathrm{b}}$ genes respectively, let us assume these genes are ordered $\mathrm{a}_{1}, \mathrm{a}_{2}, \ldots, \mathrm{a}_{\mathrm{Na}}$ and $\mathrm{b}_{1}$, $b_{2}, \ldots, b_{N b}$ respectively. Then, two genes $a_{i}$ and $b_{j}\left(1<i<N_{a^{\prime}}\right.$ $1<\mathrm{j}<\mathrm{N}_{\mathrm{b}}$ ) are defined as GCO when the following two conditions are met: (1) $a_{i}, b_{j}$ exhibit statistically significant sequence similarity; (2) at least one of $\mathrm{a}_{\mathrm{i}-1}, \mathrm{a}_{\mathrm{i}+1}$ exhibits statistically significant sequence similarity with one of $b_{j-1}$, $\mathrm{b}_{\mathrm{j}+1}$.

\section{Genome comparison}

We applied a relatively strict E-value cut-off $\left(\mathrm{E} \leq 10^{-6}\right)$ and an additional requirement of 50\% length coverage of both query and target sequences by the BLAST alignment. This approach was chosen in order to avoid spurious matches due to short conserved sequence motifs. The same criteria were applied both in the cross-genome comparison and in the self-comparison as presented in text. Differences from the results reported in [2] originate from the fact that we have used the latest versions of the E. coli K12 and Salmonella genomes, the difference in the E-value cut-off and the introduction of the alignment coverage criterion in this work.

\section{Ka/Ks estimation}

We used the pairwise alignments reported by BLASTP to guide the construction of alignments of the corresponding coding regions, inserting in-frame gaps where necessary. For the $\mathrm{Ka} / \mathrm{Ks}$ ratio calculation we used the yn00 program of the PAML package $[25,26]$ with the following parameters:

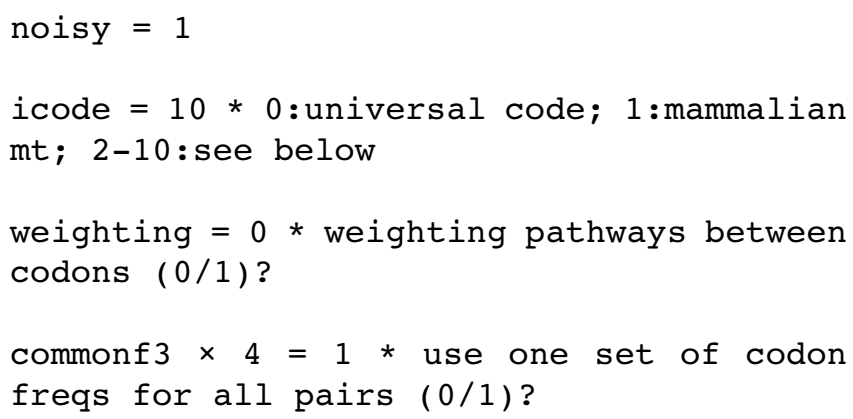


We have removed two genes (NP_462323 and NP_463455) for which the program couldn't provide us with a reliable $\mathrm{Ka} / \mathrm{Ks}$ ratio, as well as all genes with $\mathrm{Ka} / \mathrm{Ks}$ $=0$ (Additional file 7). In order to avoid probable cases of saturated Ks values, where the estimated $\mathrm{Ka} / \mathrm{Ks}$ ratio may be unreliable, we have further filtered our dataset to contain only those homologs with Ks $<1.5$ according to [37]. Nevertheless, since sequences of nGCO genes on average seem to accumulate more non-synonymous substitutions than those of GCO genes, the difference in the $\mathrm{Ka} / \mathrm{Ks}$ ratio between GCO and nGCO genes would probably be higher than the one reported here.

\section{Statistical analysis}

All statistical calculations have been performed with $\mathrm{R}$ (Version 2.3.1) R Development Core Team. R: A language and environment for statistical computing. R Foundation for Statistical Computing, Vienna, Austria, 2004. URL http://www.R-project.org. 3-900051-07-0.

\section{Competing interests}

The authors declare that they have no competing interests.

\section{Authors' contributions}

II and VJP conceived and designed the experiments. NP, KT and II collected, cleansed and reformatted data. NP, KT, VJP and II performed the experiments. TT performed Statistical Analysis. II and VJP Wrote the paper. All authors read and approved the final manuscript.

\section{Additional material}

\section{Additional file 1}

List of Salmonella and E. coli homologs. A table containing information about Salmonella and $\mathrm{E}$. coli homologs.

Click here for file

[http://www.biomedcentral.com/content/supplementary/1471-

2164-10-597-S1.DOC]

\section{Additional file 2}

GC content analysis of the Salmonella gene set after removing predicted HTGs. Table displaying the content analysis of the Salmonella gene set after removing predicted HTGs.

Click here for file

[http://www.biomedcentral.com/content/supplementary/14712164-10-597-S2.DOC]

\section{Additional file 3}

Duplicates in Salmonella that share the same best hit in E. coli K12. Table displaying in Salmonella that share the same best hit in $\mathrm{E}$. coli K12.

Click here for file

[http://www.biomedcentral.com/content/supplementary/14712164-10-597-S3.DOC]

\section{Additional file 4}

List of Salmonella $n G C O$ duplicated genes. Table displaying of Salmonella $n G C O$ duplicated genes.

Click here for file

[http://www.biomedcentral.com/content/supplementary/1471-

2164-10-597-S4.DOC]

\section{Additional file 5}

Genes with no homolog between Salmonella and E. coli k12 that share a homolog between Salmonella and EPEC. Table displaying Genes with no homolog between Salmonella and E. coli k12 that share a homolog between Salmonella and EPEC.

Click here for file

[http://www.biomedcentral.com/content/supplementary/14712164-10-597-S5.DOC]

\section{Additional file 6}

Salmonella genes whose order switches from nGCO to GCO when the reference genome is $K 12$ and EPEC, respectively. Table displaying Salmonella genes whose order switches from nGCO to GCO when the reference genome is $K 12$ and EPEC, respectively.

Click here for file

[http://www.biomedcentral.com/content/supplementary/1471-

2164-10-597-S6.DOC]

\section{Additional file 7}

Salmonella genes that were excluded from $\mathrm{Ka} / \mathrm{Ks}$ ratio calculations. Table displaying Salmonella genes that were excluded from $\mathrm{Ka} / \mathrm{Ks}$ ratio calculations.

Click here for file

[http://www.biomedcentral.com/content/supplementary/14712164-10-597-S7.DOC]

\section{Additional file 8}

Gene order conservation of H-NS repressed genes predicted to be HTGs is not associated to their GC content. We illustrate features of the subset of $130 \mathrm{H}$-NS repressed genes predicted to be HTGs (HNS-HTGs). More specifically, the distribution of HNS-HTGs genes in the three categories depending on gene order conservation largely deviates from the overall genome representation, with a striking under-representation of GCO genes. Additionally, the average GC content between these gene sets does not exhibit significant differences among the three gene classes (KruskalWallis chi-squared $=1.1224, d f=2, P$-value $<0.7717)$. This finding was expected since the prediction of the HTGs is mainly based on GC content (Garcia-Vallve S, Guzman E, Montero MA, Romeu A: HGT-DB: a database of putative horizontally transferred genes in prokaryotic complete genomes. Nucleic Acids Res 2003, 31(1):187-189.)

Click here for file

[http://www.biomedcentral.com/content/supplementary/14712164-10-597-S8.DOC]

\section{Additional file 9}

Correlation of gene order conservation with poisson and gamma corrected distances for multiple substitutions. Poisson and gamma corrected distances for multiple substitutions were calculated as described in Yang Z., "Computational Molecular Evolution", Oxford University Press, 2006 ( $p p .45$ and 46 respectively).

Click here for file

[http://www.biomedcentral.com/content/supplementary/14712164-10-597-S9.DOC] 


\section{Additional file 10}

A detailed legend of Fig. 1including statistical analysis. A Word DOC containing a full legend for figure 1 .

Click here for file

[http://www.biomedcentral.com/content/supplementary/14712164-10-597-S10.DOC]

\section{Acknowledgements}

We thank Christos Ouzounis (Kings College, London), Pantelis Georgiades (University of Cyprus) and loannis Kyrmitzoglou (University of Cyprus) for critically reading of the manuscript, useful discussions and suggestions. Funding to pay the Open Access publication charges for this article was provided by the University of Cyprus. We thank Mpriki for great inspiration. This paper is dedicated to the memory of Prof. Costas Kastritsis.

\section{References}

I. Dandekar T, Snel B, Huynen M, Bork P: Conservation of gene order: a fingerprint of proteins that physically interact. Trends Biochem Sci 1998, 23(9):324-328.

2. Theodosiou T, Iliopoulos I: Protein sequences of linked genes are highly conserved in two bacterial species. J Evol Biol 2006, 19(4): 1343-1345.

3. Cohen BA, Mitra RD, Hughes JD, Church GM: A computational analysis of whole-genome expression data reveals chromosomal domains of gene expression. Nat Genet 2000, 26(2): $183-186$.

4. Williams EJ, Hurst LD: The proteins of linked genes evolve at similar rates. Nature 2000, 407(6806):900-903.

5. Pal C, Hurst LD: Evidence for co-evolution of gene order and recombination rate. Nat Genet 2003, 33(3):392-395.

6. Jordan IK, Rogozin IB, Wolf YI, Koonin EV: Essential genes are more evolutionarily conserved than are nonessential genes in bacteria. Genome Res 2002, I 2(6):962-968.

7. Fraser HB, Hirsh AE, Steinmetz LM, Scharfe C, Feldman MW: Evolutionary rate in the protein interaction network. Science 2002 , 296(5568):750-752.

8. Drummond DA, Bloom JD, Adami C, Wilke CO, Arnold FH: Why highly expressed proteins evolve slowly. Proc Natl Acad Sci USA 2005, I 02(40): $14338-14343$.

9. Pal C, Papp B, Hurst LD: Highly expressed genes in yeast evolve slowly. Genetics 200I, I58(2):927-93I.

10. Ohno S: Evolution by gene duplication. Berlin, New York: Springer-Verlag; 1970.

II. Notebaart RA, Huynen MA, Teusink B, Siezen RJ, Snel B: Correlation between sequence conservation and the genomic context after gene duplication. Nucleic Acids Res 2005, 33(19):6164-6171.

12. Lucchini S, Rowley G, Goldberg MD, Hurd D, Harrison M, Hinton IC: $\mathrm{H}$-NS mediates the silencing of laterally acquired genes in bacteria. PLoS Pathog 2006, 2(8):e8।

13. Navarre WW, Porwollik S, Wang Y, McClelland M, Rosen H, Libby SJ, Fang FC: Selective silencing of foreign DNA with low GC content by the H-NS protein in Salmonella. Science 2006, 313(5784):236-238.

14. Dorman C]: H-NS: a universal regulator for a dynamic genome. Nat Rev Microbiol 2004, 2(5):39|-400.

15. Dorman CJ: H-NS, the genome sentinel. Nat Rev Microbiol 2007, 5(2): $157-16 \mid$.

16. Pflum MK: H-NS gives invading DNA the silent treatment. Nat Chem Biol 2006, 2(8):400-40I.

17. Lawrence JG, Ochman H: Amelioration of bacterial genomes: rates of change and exchange. I Mol Evol I997, 44(4):383-397.

18. Medigue C, Rouxel T, Vigier P, Henaut A, Danchin A: Evidence for horizontal gene transfer in Escherichia coli speciation. J Mol Biol 199I, 222(4):85I-856.

19. McClelland M, Sanderson KE, Spieth J, Clifton SW, Latreille P, Courtney L, Porwollik S, Ali J, Dante M, Du F, et al:: Complete genome sequence of Salmonella enterica serovar Typhimurium LT2. Nature 200I, 4I3(6858):852-856.
20. Blattner FR, Plunkett G, Bloch CA, Perna NT, Burland V, Riley M, Collado-Vides J, Glasner JD, Rode CK, Mayhew GF, et al:: The complete genome sequence of Escherichia coli K-12. Science 1997, 277(533I): | 1453-| 474 .

21. Altschul SF, Madden TL, Schaffer AA, Zhang J, Zhang Z, Miller W, Lipman DJ: Gapped BLAST and PSI-BLAST: a new generation of protein database search programs. Nucleic Acids Res 1997, 25(I7):3389-3402.

22. Garcia-Vallve S, Guzman E, Montero MA, Romeu A: HGT-DB: a database of putative horizontally transferred genes in prokaryotic complete genomes. Nucleic Acids Res 2003, 31 (I): 187-189.

23. Coulondre C, Miller JH, Farabaugh PJ, Gilbert W: Molecular basis of base substitution hotspots in Escherichia coli. Nature 1978, 274(5673): $775-780$.

24. Rocha EP, Danchin A: Base composition bias might result from competition for metabolic resources. Trends Genet 2002, I 8(6):291-294.

25. Yang Z, Nielsen R: Estimating synonymous and nonsynonymous substitution rates under realistic evolutionary models. Mol Biol Evol 2000, 17(1):32-43.

26. Yang Z: PAML: a program package for phylogenetic analysis by maximum likelihood. Comput Appl Biosci 1997, I3(5):555-556.

27. Kondrashov FA, Rogozin IB, Wolf YI, Koonin EV: Selection in the evolution of gene duplications. Genome Biol 2002, 3(2):RESEARCH0008.

28. Knuth $\mathrm{K}$, Niesalla H, Hueck CJ, Fuchs TM: Large-scale identification of essential Salmonella genes by trapping lethal insertions. Mol Microbiol 2004, 5 I (6): I 729 - I 744.

29. Iguchi A, Thomson NR, Ogura Y, Saunders D, Ooka T, Henderson IR, Harris D, Asadulghani M, Kurokawa K, Dean P, et al.: Complete genome sequence and comparative genome analysis of enteropathogenic Escherichia coli O127:H6 strain E2348/69. J Bacteriol 2009, I 9 I (I):347-354.

30. Hensel M, Shea JE, Raupach B, Monack D, Falkow S, Gleeson C, Kubo $T$, Holden DW: Functional analysis of ssaJ and the ssaK/U operon, I 3 genes encoding components of the type III secretion apparatus of Salmonella Pathogenicity Island 2. Mol Microbiol I997, 24(I): I55-167.

31. Ruiz-Albert J, Mundy R, Yu XJ, Beuzon CR, Holden DW: SseA is a chaperone for the SseB and SseD translocon components of the Salmonella pathogenicity-island-2-encoded type III secretion system. Microbiology 2003, I49(Pt 5): I I03-I III.

32. Fass $E$, Groisman EA: Control of Salmonella pathogenicity island-2 gene expression. Curr Opin Microbiol 2009, I 2(2):199-204.

33. Batada NN, Hurst LD, Tyers M: Evolutionary and physiological importance of hub proteins. PLoS Comput Biol 2006, 2(7):e88.

34. Brown $K R$, Jurisica I: Unequal evolutionary conservation of human protein interactions in interologous networks. Genome Biol 2007, 8(5):R95.

35. Jordan IK, Wolf YI, Koonin EV: No simple dependence between protein evolution rate and the number of protein-protein interactions: only the most prolific interactors tend to evolve slowly. BMC Evol Biol 2003, 3:I.

36. Navarre WW, McClelland M, Libby SJ, Fang FC: Silencing of xenogeneic DNA by $\mathrm{H}-\mathrm{NS}$-facilitation of lateral gene transfer in bacteria by a defense system that recognizes foreign DNA. Genes Dev 2007, 2 I(I 2): I 456-I 47I.

37. Castillo-Davis $\mathrm{Cl}$, Hartl DL, Achaz G: cis-Regulatory and protein evolution in orthologous and duplicate genes. Genome Res 2004, I 4(8): 1530-1536.

Publish with BioMed Central and every scientist can read your work free of charge

"BioMed Central will be the most significant development for disseminating the results of biomedical research in our lifetime. "

Sir Paul Nurse, Cancer Research UK

Your research papers will be:

- available free of charge to the entire biomedical community

- peer reviewed and published immediately upon acceptance

- cited in PubMed and archived on PubMed Central

- yours - you keep the copyright 